\title{
Epidemiology of Candida albicans and non-C.albicans of neonatal candidemia at a tertiary care hospital in western China
}

\author{
Jinjian $\mathrm{Fu}^{1 \dagger}$, Yanling Ding ${ }^{1 \dagger}$, Ba Wei ${ }^{2 \dagger}$, Lin Wang ${ }^{3 \dagger}$, Shaolin Xu , Peixu Qin ${ }^{1}$, Liuhua Wei ${ }^{4^{*}}$ and Lijun Jiang ${ }^{4^{*}}$
}

\begin{abstract}
Background: Although the majority of Candida infections occur in the developing world, candidemia epidemiology is poorly understood in these countries. The aim of this study was to investigate the epidemiology of non-Candida albicans (non-C. albicans) candidemia among neonates at Liuzhou Maternity and Child Healthcare Hospital in China.

Methods: A retrospective review of all positive blood culture about Candida species in neonatal intensive care unit was conducted between January 2012 and November 2015. Information about demographics, risk factors and outcome of candidemia were collected. Univariate and multivariate logistic regression models were used to identify the risk factors associated with the development of non-C.albicans candidemia.

Results: The prevalence of candidemia in infants was 1.4\%. Non-C.albicans was responsible for $56.5 \%$ of neonatal candidemia. The predisposing factors for development of non-C.albicans candidemia among infants included mechanical ventilation [odds ratio (OR), 95\% confidence interval $(95 \% \mathrm{Cl})=3.13,1.07-9.14 ; P=0.037$ ] and use of assisted reproductive technology $(\mathrm{OR}, 95 \% \mathrm{Cl}=4.52,1.39-14.77 ; P=0.012)$. The overall mortality rate of candidemia was $8.7 \%$ and non-C.albicans attributed to $83.3 \%$ of all mortalities.

Conclusions: Non-C.albicans species are the major cause of candidemia in local neonatal group. The study highlights the urgent needs to evaluate the possibility of development of non-C.albicans candidemia in neonates exposed to these risk factors and much emphasis must be laid on the early implementation of medical intervention to reduce the incidences of candidemia in neonates.
\end{abstract}

Keywords: Infants, Candidemia, Non-C.albicans, Assisted reproductive technology

\section{Background}

Candida species are the third most common pathogens that attributed to neonatal bloodstream infections and are associated with 20-34\% mortality [1, 2]. Although Candida albicans has long been the most common cause of candidemia infections obtained from neonatal intensive care units (NICUs) [3, 4]. Non-C.albicans species such as Candida glabrata (C.glabrata), Candida tropicalis (C.tropicalis), Candida parapsilosis (C.parapsilosis) and Candida krusei (C.krusei) have emerged as the second or even the predominant pathogens that caused neonatal candidemia in some local hospitals [5].

\footnotetext{
*Correspondence: weiliuhua2005@163.com; jj|zgy@126.com

${ }^{\dagger}$ Equal contributors

${ }^{4}$ Department of Laboratory, Liuzhou Worker's Hospital, No 1 Liushi Rd,

Liuzhou 545005, China

Full list of author information is available at the end of the article
}

Infants are more vulnerable to develop candidemia due to their critical underlying conditions, immature immune system, and invasive operations [6, 7]. The predisposing factors contributing to the current increase of neonatal candidemia included prematurity, very low birth weight, aggressive broad-spectrum antibiotics, prolonged hospitalization, invasive operations such as central venous catheters, intubation and total parenteral nutrition [8-12].

As the Candida species formed the biofilms on the surface of indwelling medical appliances, their high virulence, the documented horizontal nosocomial transmission, vertically from mother to neonate, and their developmental of resistance to azole antifungal agents $[8,9,13]$, the neonatal candidemia has presented a challenge to pediatricians. 
Although several studies had reported the epidemiology of Candida infections in China, they mainly focused on adults, invasive Candida infections or conducted among special groups [14-17]. The investigation about Candida albicans (C.albicans) compared to non-C.albicans candidemia in neonatal groups are limited in China [18]. It is important to know local information about candidemia infections in NICUs as the Candida infections at local levels could provide pediatricians the full information to implement early medical practice to diagnosis and treat these high-risk groups. This retrospective study aimed to investigate the epidemiology of candidemia in neonatal groups at a single center in western China.

\section{Methods}

\section{Study design and data collection}

This retrospective study was conducted between January 2012 and November 2015, in NICU of Liuzhou Maternity and Child Healthcare Hospital. The medical charts for 5075 admissions during the 4year period were reviewed. Infants with positive blood culture results of Candida species were defined as candidemia. Collected demographics on previously identified risk included necrotizing enterocolitis, neurodevelopmental impairment, maternal underlying diseases (such as preeclampsia, gestational diabetes, pregnancy-induced hypertension, cholestasis, hyperthyroidism, hypothyroidism, etc.), respiratory distress, vaginal birth, fetal membrane rupture, neonatal congenital diseases (such as congenital heart disease, Glucose-6Phosplate Dehydrogenase deficiency, thalassemia, etc.), abdominal surgery, mechanical ventilation, central venous catheter, intubation, pulmonary active substance use, steroids use, antacid use, total parenteral nutrition, hospitalization duration, 3rd cephalosporins use, carbapenems use, vancomycin use, multiple antibiotic ( $\geq 3$ classes) use, antibiotic therapeutic duration (including the administration of antibiotics prior to the occurrence of candidemia and the prophylaxis antibiotic use during hospitalization), use of prophylaxis antifungal agent (fluconazole), and antifungal therapeutic duration. The collection data from medical chart was permitted by the Institutional Review Board.

\section{Microbiologic methods}

Blood samples were cultured in the BacT/AlerT 3D system (Biomérieux). Chromo Agar medium was used for Candida species identification (Biomérieux) and API 20C AuX (Biomérieux) was used for confirmation.

\section{Statistical analysis}

SPSS version 20.0 statistical software (SPSS Inc., Chicago, Il, USA) was used. Potential risk factors associated with increased candidemia risk were identified using univariate logistic regression analysis. Variables with 2-tailed $P<0.05$ were included in the multivariate logistic regression model to evaluate the odds ratios (ORs) and 95\% confidence intervals (CIs) to calculate the strength of any association. A $P$-value less than 0.05 was considered indicative of statistical significance.

\section{Results \\ The distribution of Candida species and incidence of candidemia}

There were 5075 admissions of neonates (aged $<28$ days) in the NICU, 69 infants had Candida bloodstream infections during the 4-year study period. The incidence of candidemia was $1.4 \%$. C.albicans was the leading causative pathogen of candidemia and it was isolated in $43.5 \%$ of the patients. Non-C.albicans was responsible for $56.5 \%$ of neonatal candidemia. The distribution of non-C.albicans species was C.glabrata for $33.3 \%$, C.tropicalis for $20.3 \%$, C.parapsilosis for $1.4 \%$ and C.kefyr for $1.4 \%$.

\section{Risk factors associated with candidemia due to non- C.Albicans}

Infant demographics and risk factors for candidemia are shown in Table 1. There was no difference between C.albicans and non-C.albicans in terms of the gestational age (31.7 vs 31.5 weeks, $P=0.765$ ), birth weight (1670.0 g vs $1417.6 \mathrm{~g}, P=0.128)$, or male gender $(16.7 \%$ vs $30.8 \%, P=0.150$ ).

In the univariate logistic regression model, mechanical ventilation was significantly more common in neonates with candidemia caused by non-C.albicans $(74.4 \%$ vs $46.7 \%, P=0.021)$. The antibiotic therapeutic duration was longer in the non-C.albicans group compared to C.albicans group (38.0 vs 28.1 days, $P=0.033$ ). Among infants with candidemia, $48.7 \%$ were conceived using assisted reproductive technology, compared with $16.7 \%$ in C.albicans group $(P=0.008)$.

Based on step-wise multivariate logistic regression analysis, infants with non-C.albicans candidemia infections were more likely to undergo mechanical ventilation (OR, 95\%CI $=3.13,1.07-9.14, P=0.037$ ). Infants who were conceived using assisted reproductive technology were more likely to develop non-C.albicans candidemia (OR, 95\%CI $=4.52$, 1.39-14.77, $P=0.012)$ (Table 2).

\section{Outcome}

The overall mortality among affected infants was $5.8 \%$. C.albicans and non-C.albicans were associated with a mortality rate of $3.3 \%, 12.8 \%$, respectively. There was no significance between the two groups $(P=0.197)$. 
Table 1 Clinical characteristics of neonates between C.albicans and non-C.albicans

\begin{tabular}{|c|c|c|c|c|}
\hline Variable & $\begin{array}{l}\text { C.albicans mean }(95 \% \mathrm{Cl}) \\
\text { or } \mathrm{n}(\%)\end{array}$ & $\begin{array}{l}\text { non-C.albicans mean } \\
(95 \% \text { Cl) or } n(\%)\end{array}$ & $P$ value & $\begin{array}{l}\text { odds ratio } \\
\text { (OR) }(95 \% \mathrm{Cl})\end{array}$ \\
\hline \multicolumn{5}{|l|}{ Demographics } \\
\hline gestational age (wks) & $31.7(27.6,35.8)$ & $31.5(27.7,36.3)$ & 0.765 & \\
\hline birth weight (g) & $1670.0(962.1,2377.9)$ & $1417.6(777.1,2058.1)$ & 0.128 & \\
\hline male gender, n (\%) & $22(73.3)$ & $22(56.4)$ & 0.150 & $0.47(0.17-1.32)$ \\
\hline admission age & $2.1(2.0,6.2)$ & $1.1(0.6,1.6)$ & 0.138 & \\
\hline \multicolumn{5}{|l|}{ Risk factors } \\
\hline necrotizing enterocolitis & $5(16.7)$ & $12(30.8)$ & 0.183 & $2.22(0.69-7.21)$ \\
\hline neurodevelopmental impairment & $7(23.3)$ & $8(20.5)$ & 0.778 & $0.85(0.27-2.68)$ \\
\hline maternal underlying diseases & $12(40.0)$ & $18(46.2)$ & 0.609 & $1.29(0.50-3.36)$ \\
\hline assisted reproductive technology & $5(16.7)$ & $19(48.7)$ & 0.008 & $4.75(1.51-14.96)$ \\
\hline vaginal birth & $19(63.3)$ & $23(59.0)$ & 0.713 & $1.20(0.45-3.20)$ \\
\hline fetal membrane rupture (h) & $16.4(28.4,61.2)$ & $26.6(36.0,89.2)$ & 0.459 & \\
\hline congentital diseases & $13(43.3)$ & $24(61.5)$ & 0.135 & $2.09(0.80-5.51)$ \\
\hline abdominal surgery & $4(13.3)$ & $6(15.4)$ & 0.811 & $1.18(0.30-4.63)$ \\
\hline mechanical ventilation & $14(46.7)$ & $29(74.4)$ & 0.021 & $3.31(1.20-9.15)$ \\
\hline central venous catheter & $14(46.7)$ & $27(69.2)$ & 0.061 & $2.57(0.96-6.91)$ \\
\hline intubation & $10(33.3)$ & $21(53.8)$ & 0.092 & $2.33(0.87-6.26)$ \\
\hline pulmonary active substance use & $7(23.3)$ & $9(23.1)$ & 0.980 & $0.99(0.32-3.04)$ \\
\hline steroids use & $6(20.0)$ & $9(23.1)$ & 0.759 & $1.20(0.38-3.84)$ \\
\hline antacid use & $3(10.3)$ & $8(21.1)$ & 0.250 & $2.31(0.56-9.63)$ \\
\hline total parenteral nutrition & $23(76.7)$ & $36(92.3)$ & 0.080 & $3.65(0.86-15.57)$ \\
\hline hospitalization duration (d) & $43.8(19.1,78.5)$ & $49.1(26.6,71.6)$ & 0.367 & \\
\hline $3^{\text {rd }}$ cephalosporins use & $16(55.2)$ & $23(59.0)$ & 0.754 & $1.17(0.44-3.08)$ \\
\hline carbapenems use & $21(70.0)$ & $30(76.9)$ & 0.517 & $1.43(0.49-4.20)$ \\
\hline vancomycin use & $4(13.3)$ & $6(15.4)$ & 0.811 & $1.18(0.30-4.63)$ \\
\hline multiple antibiotic use & $19(63.3)$ & $18(46.2)$ & 0.158 & $0.50(0.19-1.31)$ \\
\hline antibiotic therapeutic duration (d) & $28.1(10.0,46.2)$ & $38.0(19.3,56.7)$ & 0.033 & \\
\hline prophylaxis antifungal therapy & $19(63.3)$ & $32(82.1)$ & 0.084 & $2.65(0.88-7.99)$ \\
\hline antifungal therapeutic duration (d) & $7.9(2.3,13.5)$ & $8.8(2.3,15.3)$ & 0.535 & \\
\hline \multicolumn{5}{|l|}{ Outcome } \\
\hline death & $1(3.3)$ & $5(12.8)$ & 0.197 & $4.27(0.47-38.62)$ \\
\hline
\end{tabular}

\section{Discussion}

Candida species have emerged as important pathogens which are associated with significant morbidity and mortality in neonates $[1,10]$. In the past two decades, there have been several cohort studies indicating that the cause of Candida bloodstream infection has shifted from C.albicans to non-C.albicans [19-21]. Widespread use of azole antifungal agents, especially fluconazole, was one

Table 2 Multivariate analysis for C.albicans and non-C.albicans

\begin{tabular}{llll}
\hline Risk factor & Odds ratio & $95 \% \mathrm{Cl}$ & $P$ value \\
\hline assisted reproductive technology & 4.52 & $1.39-14.77$ & 0.012 \\
mechanical ventilation & 3.13 & $1.07-9.14$ & 0.037 \\
\hline
\end{tabular}

of the reasons contributing to the increased isolation rate of non-C.albicans and the decreased isolation rate of C.albicans [22, 23]. Many studies from western countries showed that C.albicans was the predominant Candida species isolated from candidemia, followed by C.parapsilosis among infants $[3,22]$. It was reported that C.parapsilosis attributed to one-fifth of all cases of candidemia in neonates [24]. Our study revealed that C.parapsilosis attributed to only $1.4 \%$ of all cases of neonatal candidemia. In our study, we observed a changing pattern of neonatal candidemia among infant patients. The most frequent Candida species isolated from the bloodstream was C.albicans (43.5\%). The most common non-C.albicans species was C.glabrata (33.3\%), followed 
by C.tropicalis (20.3\%). The non-C.albicans together accounted for more than $55 \%$ of all candidemia in our center. This species distribution pattern varied from western countries but was consistent with studies conducted in Australia and in 11 NICUs in China [21, 25]. It was reported that the difference of prevalence of candidemia due to a specific Candida specie may vary by geographic region [15], and some researches recommended that the study should focus at the local level rather than at the worldwide scale [26]. One of the outbreak of candidemia caused by C.parapsilosis reported from a Chinese hospital revealed that all strains was susceptible to fluconazole [27]. Fluconazole has been recommended as the best alternative antifungal drug for both of prophylaxis and primary treatment for patients with candidemia due to its efficacy and safety [28, 29]. In China, C.parapsilosis isolated from sterile body fluids have rarely been reported to be resistant to fluconazole [30, 31]. A prospective cohort study from a teaching hospital conducted between 2006 and 2011 reported that all 25 C.parapsilosis strains tested were susceptible to fluconazole [30]. The 2010 National China Hospital Invasive Fungal Surveillance Net (CHIFNET) program found that only $1.4 \%$ of C.parapsilosis strains were resistant to fluconazole [31]. Based on those studies, we can state with certainty that C.parapsilosis specie isolated from blood stream is still highly susceptible to fluconazole in Chinese region and the use of fluconazole for prophylaxis in our hospital can be effective.

Many investigations have confirmed that use of broadspectrum antibiotics and prolonged antibiotic therapeutic duration were the most common risk factors associated with neonatal candidemia [32]. A previous study showed that prolonged use of 3rd generation cephalosporin was associated with neonatal candidemia due to C.parapsilosis in infants [33]. Previous studies also suggested that prolonged antibiotic therapeutic duration predisposed to C.glabrata was attributed to candidemia [34, 35]. Our observation was in agreement with the general view that prolonged antibiotic use was associated with an increase of non-C.albicians candidemia in patients. It was reported that universal exposure to antibacterial drugs would suppress the bacterial flora and facilitate Candida colonization, which may increase the potential risk of development of candidemia [32]. These findings highlight the importance of evaluating the antibacterial burden in infected neonates.

In the multivariate logistic regression models, we found that mechanical ventilation affected the risk of candidemia infection with non-C.albicans isolates. It is well known that mechanical ventilation would play an important role in the pathogenesis of invasive candidiasis because of abrading the respiratory mucosa, providing Candida species for a portal of entry into the bloodstream [32]. Once Candida species adheres to the surface of invasive medical devices, it can form a biofilm thus protecting itself from immune responses and antifungal therapy.

Additionally, our analysis identified that use of assisted reproductive technology (ART) such as in vitro fertilization-assisted pregnancy would serve as an independent risk factor for development of nonC.albicans candidemia in newborns (OR, 95\% $C I=4.52,1.39-14.77, P=0.012)$. This association may be partly due to the high proportion infection caused by C.glabrata among the neonates whom were the production of ART. In the non-C.albicans candidemia group, $73.7 \%(14 / 19)$ of neonates were conceived using ART. Globally, few studies have verified the association of ART and the outcome of neonatal candidemia. Most of the previous literature focused on the Candida species was associated with chorioamnionitis in in vitro fertilization (IVF) pregnancy [36]. It was reported that some types of uterine manipulation, such as IVF, may introduce the fungus at the time of embryo transfer [37]. Although C.glabrata showed a reduced virulence and absence of pathognomonic lesions compared with C.albicans, there was strong documented evidence of association between chorioamnionitis caused by C.glabrata and the use of ART, and the high lethality and morbidity in such group [38]. One of the studies showed that in $72 \%$ of cases of C.glabrata chorioamnionitis, conception was through ART [39]. These results raised the concern of the need for Candida species screening prior to IVF initialization. Although we failed to find out the data of culture results before embryo transfer, $73.9 \%$ of the infected neonates had received prophylaxis antifungal agents (fluconazole). Among the patients who were conceived using ART, 91.7\% cases were administered fluconazole for prophylactic use. This may partly explain why the mortality rate was lower (overall mortality rate was $8.7 \%$ ) that previous reports (24-30\% in neonatal candidemia) [32].

The main limitation of our study was the retrospective design and the small sample size, which may compromise the statistical power. Additionally, we cannot collect the data of maternal yeasts colonization before and after delivery, which may not provide the enough information for evaluation of the vertical transmission and the association about maternal yeasts colonization and the subsequence of development of neonatal candidemia. Nevertheless, our data revealed that prolonged antibiotic therapeutic duration, mechanical ventilation and use of assisted reproductive technology were all the independent risk 
factors for the development of neonatal non-C.albicans candidemia.

\section{Conclusions}

Non-C.albicans species are the major cause of candidemia in local neonatal group. The study highlights the urgent needs to evaluate the possibility of development of non-C.albicans candidemia in neonates exposed to these risk factors and much emphasis must be laid on the early implementation of medical intervention to reduce the incidences of candidemia in neonates.

\section{Abbreviations}

95\%Cls: 95\% confidence intervals; ART: Assisted reproductive technology; C.albicans: Candida albicans; C.glabrata: Candida glabrata; C.krusei: Candida krusei; C.parapsilosis: Candida parapsilosis; C.tropicalis: Candida tropicalis; CHIFNET: China Hospital Invasive Fungal Surveillance Net; IVF: in vitro fertilization; NICUs: Neonatal intensive care units; non-C. albicans: non-Candida albicans; ORs: Odds ratios

\section{Acknowledgements}

Not applicable.

\section{Funding}

None.

\section{Availability of data and materials}

The datasets generated and/or analyzed during the current study are not publicly available due to the confidentiality permission with the Institutional Review Board but are available from the corresponding author on reasonable request.

\section{Authors' contributions}

YD and $L J$ designed the study and drafted an outline. JF, YD, BW, LW, SX, PQ, $L W$ and $L J$ participated in data analysis, JF drafted the manuscript and revised it critically for important intellectual content. All of authors approved the final content off this manuscript.

\section{Competing interests}

The authors declare that they have no competing interests.

\section{Consent for publication}

Not applicable.

\section{Ethics approval and consent to participate}

This study was approved by the Institutional Review Board of Liuzhou Maternity and Child Healthcare Hospital.

\section{Publisher's Note}

Springer Nature remains neutral with regard to jurisdictional claims in published maps and institutional affiliations.

\section{Author details \\ ${ }^{1}$ Department of Laboratory, Liuzhou Maternity and Child Healthcare Hospital, Liuzhou, China. ${ }^{2}$ Department of Neonatology, Liuzhou Maternity and Child Healthcare Hospital, Liuzhou, China. ${ }^{3}$ Department of Science and Education, Liuzhou Maternity and Child Healthcare Hospital, Liuzhou, China. \\ ${ }^{4}$ Department of Laboratory, Liuzhou Worker's Hospital, No 1 Liushi Rd, Liuzhou 545005, China.}

Received: 10 October 2016 Accepted: 26 April 2017

Published online: 06 May 2017

\section{References}

1. Benjamin DK Jr, Stoll BJ, Fanaroff AA, McDonald SA, et al. National Institute of Child Health and Human Development neonatal research network. Neonatal candidiasis among extremely low birth weight infants: risk factors, mortality rates, and neurodevelopmental outcomes at 18 to 22 months. Pediatrics. 2006:117:84-92.

2. Shetty SS, Harrison LH, Hajjeh RA, Taylor T, et al. Determining risk factors for candidemia among newborn infants from population-based surveillance: Baltimore, Maryland, 1998-2000. Pediatr Infect Dis J. 2005;24:601-4.

3. Oeser C, Lamagni T, Heath PT, Sharland M, et al. The epidemiology of neonatal and pediatric candidemia in England and Wales, 2000-2009. Pediatr Infect Dis J. 2013;32:23-6.

4. Steinbach WJ, Roilides E, Berman D, Hoffman JA, et al. Results from a prospective, international, epidemiologic study of invasive candidiasis in children and neonates. Pediatr Infect Dis J. 2012:31:1252-7.

5. Trofa D, Gácser A, Nosanchuk JD. Candida parapsilosis, an emerging fungal pathogen. Clin Microbiol Rev. 2008;21:606-25.

6. Filioti J, Spiroglou K, Panteliadis CP, Roilides E. Invasive candidiasis in pediatric intensive care patients: epidemiology, risk factors, management, and outcome. Intensive Care Med. 2007;33:1272-83.

7. Carey AJ, Saiman L, Polin RA. Hospital-acquired infectious in the NICU: epidemiology for the new millennium. Clin Perinatol. 2008;35:223-49.

8. Sighi S, Deep A. Invasive candidiasis in pediatric intensive care units. Indian J Pediatr. 2009;76:1033-44.

9. Roilides E. Invasive candidiasis in neonates and children. Early Hum Dev. 2011;87:S75-6

10. Benjamin DK Jr, Stoll BJ, Gantz MG, Walsh MC, et al. Neonatal candidiasis: epidemiology, risk factors, and clinical judgment. Pediatrics. 2010;126:e865-73.

11. Rodriguez D, Almirante B, Park BJ, Cuenca-Estrella M, et al. Candidemia in neonatal intensive caur units: Barcelona, Spain. Pediatr Infect Dis J. 2006;25:224-9.

12. Fridkin SK, Kaufman D, Edwards JR, Shetty S, et al. Changing incidence of Candida bloodstream infections among NICU patients in the United States: 1995-2004. Pediatrics. 2006;117:1680-7.

13. Tumbarello M, Posteraro B, Trecarichi EM, Fiori B, et al. Biofilm production by Candida species and inadequate antifungal therapy as predictors of mortality for patients with candidemia. J Clin Microbiol. 2007:45:1843-50.

14. Li C, Wang H, Yin M, Han H, et al. The differences in the epidemiology and predictors of death between candidemia acquired in intensive care units and other hospital settings. Intern Med. 2015:54:3009-16.

15. Zhang XB, Yu SJ, Yu JX, Gong YL, et al. Retrospective analysis of epidemiology and prognostic factors for candidemia at a hospital in China, 2000-2009. Jpn J Infect Dis. 2012;65:510-5.

16. Wu Z, Liu Y, Feng $X$, Liu Y, et al. Candidemia: incidence rates, type of species, and risk factors at a tertiary care academic hospital in China. Int J Infect Dis. 2014:22:4-8.

17. Ding $X$, Yan D, Sun W, Zeng Z, et al. Epidemiology and risk factors for nosocomial Non-Candida albicans candidemia in adult patients at a tertiary care hospitals in North China. Med Mycol. 2015;53:684-90.

18. Hua S, Huang J, Wu Z, Feng Z. A comparison study between Candida parapsilosis sepsis and Candida albicans sepsis in preterm infants. Turk J Pediatr. 2012;54:502-8

19. Dzierzanowska-Fangrat K, Romanowska E, Gryniewicz-Kwiatkowska O, Migdal M, et al. Candidemia in a polish tertiary paediatric hospital, 2000 to 2010. Mycoses. 2014:57:105-9.

20. VelascoE BR. A prospective cohort study evaluating the prognostic impact of clinical charaeteristics and comorbid conditions of hospitalized adult and pediatric cancer patients with candidemia. Eur J Clin Microbiol Infect Dis. 2008:27:1071-8

21. Blyth CC, Chen SC, Slavin MA, Serena C, et al. Australian Candidemia Study. Not just little adults: candidemia epidemiology, molecular characterization, and anti-fungal susceptibility in neonatal and pediatric patients. Pediatrics. 2009;123:1360-8.

22. Sendid B, Cotteau A, Francois N, D'Haveloose A, et al. Candidemia and antifungal therapy in a French university hospital: rough trends over a decade and possible links. BMC Infect Dis. 2006:6:80-8.

23. Trick WE, Fridkin SK, Edwards JR, Hajjeh RA, et al. National Nosocomial Infections Surveillance System Hospitals. Secular trend of hospital-acquired candidemia among intensive care unit patients in the United States during 1989-1999. Clin Infect Dis. 2002;35:627-30.

24. Oser C, Vergnano S, Naidoo R, Anthony M, et al. Neonatal invasive fungal infection in England 2004-2010. Clin Microbiol Infect. 2014;20:936-41.

25. Xia H, Wu H, Xia S, Zhu X, et al. Invasive candidiasis in preterm neonates in China: a retrospective study from 11 NICUS during 2009-2011. Pediatr Infect Dis J. 2014;33:106-9. 
26. Mikulsha M, Bassetti M, Ratto S, Viscoli C. Invasive candidiasis in non-hematological patients. Mediterr J Hematol Infect Dis. 2011;3:e2011007.

27. He W, Zhang L, Kudinha T, Kong F, et al. Investigation of an unrecognized large-scale outbreak of Candida parapsilosis Sensu stricto fungaemia in a tertiary-care hospital in China. Sci Rep. 2016;6:27099.

28. Pinhati HM, Casulari LA, Souza AC, Siqueira RA, et al. Outbreak of candidemia caused by fluconazole resistant Candida parapsilosis strains in an intensive care unit. BMC Infect Dis. 2016;16(1):433.

29. Pappas PG, Kauffman CA, Andes D, Benjamin DK, et al. Clinical practice guidelines for the management of candidiasis: 2009 update by the Infectious Diseases Society of America. Clin Infect Dis. 2009;48:503-35.

30. Li F, Wu L, Cao B, Zhang Y, et al. Surveillance of the prevalence, antibiotic susceptibility, and genotypic characterization of invasive candidiasis in a teaching hospital in China between 2006 to 2011. BMC Infect Dis. 2013;13:353.

31. He W, Xiao M, Chen SC-A, Kong F, et al. In vitro susceptibilities of yeast species to Fluconazole and Voriconazole as determined by the 2010 National China Hospital Invasive Fungal Surveillance net (CHIF-NET) study. J Clin Microbiol. 2012;50(12):3952-9.

32. Kelly MS, Benjamin DK Jr, Smith PB. The epidemiology and diagnosis of invasive candidiasis among premature infants. Clin Perinatol. 2015;42:105-17.

33. Benjamin DK Jr, Ross K, Mckinney RE Jr, Benjamin DK, et al. When to suspect fungal infection in neonates: a clinical comparison of Candida albicans and Candida parapsilosisfungemia with coagulase-negative staphylococcal bacteremia. Pediatrics. 2000;106:712-8.

34. Chow JK, Golan Y, Ruthazer R, Karchmer AW, et al. Risk factors for albicans and non-albicans candidemia in the intensive care unit. Cri Care Med. 2008;36:1993-8.

35. Lin MY, Carmeli Y, Zumsteg J, Flores EL, et al. Prior antimicrobial therapy and risk for hospital-acquired Candida glabrata and Candida kruseifungemia: a case-case-control study. Antimicrob Agents Chemother. 2005;49:4555-60

36. Ganer Herman H, MevorachZussman N, KrajdenHaratz K, Bar J, et al. Candida glabratachorioamnionitis following in vitro fertilization: review of the literature. Gynecol Obstet Invest. 2015;80:145-7.

37. Asemota OA, Nyirjesy P, Fox R, Sobel JD. Candida glabratacomplicating in vitro pregnancy: successful management of subsequent pregnancy. Fertil Steril. 2011;95:803. e1-2.

38. Tan SQ, Ng OT, Khong CC. Candida glabrataspesis associated with chorioamnionitis in an IVF twin pregnancy: should we deliver? J Obstet Gynaecol Res. 2015;41:962-6.

39. Alfei A, Rizzo A, Cavanna C, Lallitto F, et al. Candida Glabrata and pre-term premature rupture of membrane complicating in vitro pregnancy: case report and confirmation of mother to neonate transmission. Arch Gynecol Obset. 2014;290:211-4.

\section{Submit your next manuscript to BioMed Central and we will help you at every step:}

- We accept pre-submission inquiries

- Our selector tool helps you to find the most relevant journal

- We provide round the clock customer support

- Convenient online submission

- Thorough peer review

- Inclusion in PubMed and all major indexing services

- Maximum visibility for your research

Submit your manuscript at www.biomedcentral.com/submit

CBiomed Central 